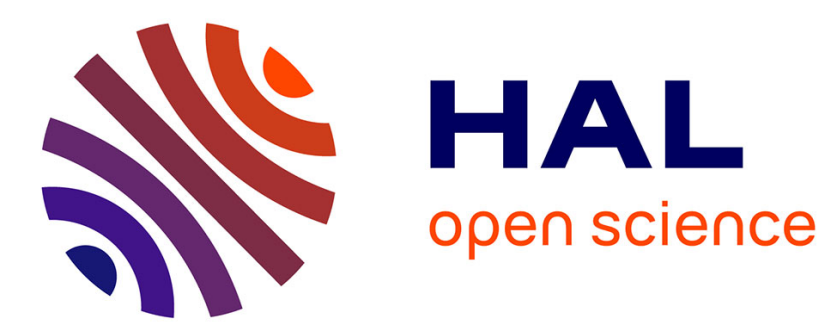

\title{
Les nouveaux verres
}

J. Lucas

\section{To cite this version:}

J. Lucas. Les nouveaux verres. Revue de Physique Appliquée, 1986, 21 (11), pp.683-687. 10.1051/rphysap:019860021011068300 . jpa-00245489

\section{HAL Id: jpa-00245489 https://hal.science/jpa-00245489}

Submitted on 1 Jan 1986

HAL is a multi-disciplinary open access archive for the deposit and dissemination of scientific research documents, whether they are published or not. The documents may come from teaching and research institutions in France or abroad, or from public or private research centers.
L'archive ouverte pluridisciplinaire HAL, est destinée au dépôt et à la diffusion de documents scientifiques de niveau recherche, publiés ou non, émanant des établissements d'enseignement et de recherche français ou étrangers, des laboratoires publics ou privés. 
Classification

Physics Abstracts

$81.20 \mathrm{P}$

\title{
Les nouveaux verres
}

\author{
J. Lucas \\ Université de Rennes, Campus de Beaulieu, Laboratoire de Chimie Minérale D, 35042 Rennes Cedex, France
}

(Reçu le 20 février 1986, accepté le 16 avril 1986)

\begin{abstract}
Résumé. - Le développement de nouveaux verres est principalement guidé par la recherche de matériaux ayant un large domaine de transmission, particulièrement dans le domaine infrarouge, et par la potentialité d'ultratransparence. Les matériaux candidats pour la fabrication de fibres optiques à très faibles pertes et de guide d'onde ayant une large fenêtre optique sont les verres de chalcogénures et les verres halogénés, en particulier les verres fluorés à base de métaux lourds dont les pertes minimales estimées sont voisines de $10^{-2} \mathrm{~dB} / \mathrm{km}$.
\end{abstract}

\begin{abstract}
The development of new glasses is mainly motivated by the search of materials having a broad transmission range, specially in the I.R. domain, and by the potential of ultratransparency. The candidates for ultralow loss optical fiber and waveguide having a large optical window are the chalcogenides and the halides glasses and among them the heavy metal fluoride glasses with estimated losses as low as $10^{-2} \mathrm{~dB} / \mathrm{km}$.
\end{abstract}

\section{Introduction.}

En raison de leur extrême importance au plan industriel, avec des applications allant de l'isolation, l'emballage, à des secteurs plus sophistiqués comme les fibres optiques, les matériaux vitreux à base de silice $\mathrm{SiO}_{2}$ dominent très largement le monde des verres [1]. D'aucuns s'étonnent même que d'autres matériaux puissent exister à l'état vitreux d'autant que, quand ceci se produit, par exemple le fluorure de béryllium $\mathrm{BeF}_{2}$ et le chlorure de zinc $\mathrm{ZnCl}_{2}$ se vitrifient facilement, ces composés sont souvent hygroscopiques, et leur vocation matériaux est sérieusement compromise.

Les oxydes de phosphore $\mathrm{P}_{2} \mathrm{O}_{5}$, oxyde de bore $\mathrm{B}_{2} \mathrm{O}_{3}$, de germanium $\mathrm{GeO}_{2}$ sont également de bons formateurs de verre et beaucoup de verres techniques résultent du fait d'associations plus ou moins complexes de ces oxydes. Le point commun de toute cette chimie est la formation d'un squelette géant apériodique qui résulte de l'association de tétraèdres élémentaires. L'adjonction d'oxydes dits modificateurs de réseau, comme $\mathrm{Na}_{2} \mathrm{O}, \mathrm{CaO}$, etc... perturbe encore plus les jonctions entre tétraèdres et abaisse les températures d'élaboration des verres. Dans tous ces verres d'oxydes, la liaison $\mathrm{M}-\mathrm{O}$ entre atomes légers $(\mathrm{M}=\mathrm{Si}, \mathrm{P}, \mathrm{B}, \mathrm{Ge})$ est très forte et il lui est associée une fréquence de vibration très haute entraînant une absorption multiphonon qui fait de ces matériaux de mauvais transmetteurs infrarouge.
La coupure I.R. est située vers $2-3 \mu \mathrm{m}$ pour les verres silicatés, phosphatés, et 3-4 $\mu \mathrm{m}$ pour les germanates.

Les recherches effectuées ces dernières années dans ce domaine des verres classiques ont moins concerné la recherche de nouveaux verres que le développement de nouvelles techniques d'élaboration comme les méthodes sol-gel qui permettent la préparation de verres spéciaux pour des applications biomédicales par exemple.

Les motivations qui justifient la recherche de nouvelles compositions vitreuses sont essentiellement de deux sortes :

1) Déplacer la frontière infrarouge vers les plus grandes longueurs d'onde en essayant d'atteindre des zones de transmision stratégiques comme la région $10,6 \mu \mathrm{m}$ qui correspond à l'émission du laser $\mathrm{CO}_{2}$.

2) Obtenir des matériaux vitreux de plus en plus transparents, voir ultratransparents pour des applications soit en technologie laser de puissance, soit pour la mise au point de fibres optiques pour télécommunications très longues distances sans répéteur.

Comme indiqué plus loin, ces deux objectifs sont d'ailleurs corrélés.

\section{Les verres pour la transmission.}

Si on envisage une composition chimique simple, du type $\mathrm{M}_{x} \mathrm{X}_{y}$, le problème posé est ici d'obtenir une 
composition vitreuse stable sachant que $M$ et $X$ doivent avoir la masse atomique la plus élevée, et que la constante de force de la liaison $\mathrm{M}-\mathrm{X}$ soit la plus petite possible.

Ces contraintes sont suffisamment sévères pour que ces conditions ne soient réunies que pour très peu de matériaux, surtout si l'on tient compte du fait que la compétition verre-cristal va dans la très grande majorité des combinaisons chimiques dans le sens de la formation de composés cristallins. La figure 1 montre comment évolue l'absorption multiphonon en fonction de la longueur d'onde pour un certain nombre de matériaux I.R., dont des verres.

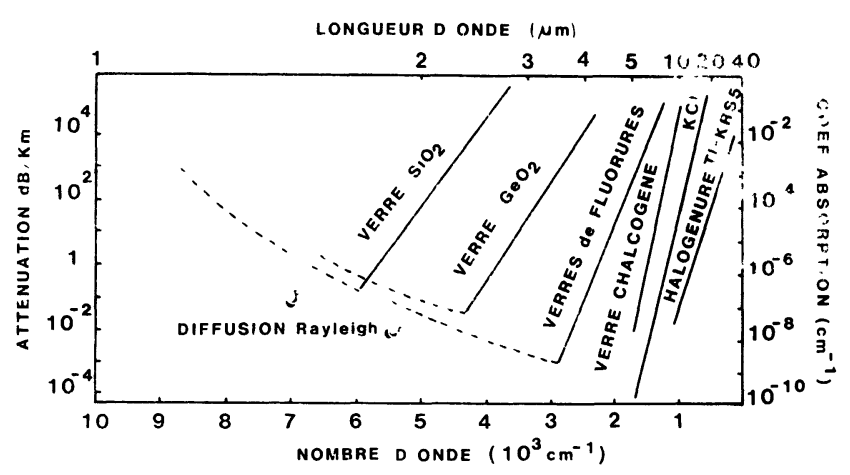

Fig. 1. - Variation de l'absorption multiphonon en fonction de la longueur d'onde pour un certain nombre de matériaux vitreux ou cristallins. L'évolution estimée de la diffusion Rayleigh en fonction de $\lambda$ est mentionnée en pointillés pour les verres bases $\mathrm{SiO}_{2}, \mathrm{GeO}_{2}$ et fluorures.

[Evolution of the multiphonon absorption versus wavelength for some crystallines and vitreous materials. The Rayleigh scattering in dotted line is mentioned for $\mathrm{SiO}_{2}, \mathrm{GeO}_{2}$ and fluoride based glasses].

Deux grandes classes de matériaux constituent l'essentiel des verres pour l'I.R. : les verres d'halogénures avec $\mathrm{X}=\mathrm{F}, \mathrm{Cl}, \mathrm{Br}$, I et les verres de chalcogènes et de chalcogénures avec $\mathrm{Y}=\mathrm{S}, \mathrm{Se}, \mathrm{Te}$, As. Cette dernière famille est étudiée depuis très longtemps pour la réalisation de pièces pour l'optique I.R., mais de nouvelles compositions ont permis de reculer significativement la frontière I.R.

Les verres d'halogénures sont par contre beaucoup plus nouveaux et les perspectives de développement technologique en ont fait un domaine de recherche très actif, ces dernières années.

2.1 LES VERRES D'HALOGÉNURES. - Toutes les familles d'halogénures [2] ont des représentants qui existent à l'état vitreux ; toutefois, les familles des chlorures, bromures, iodures conduisent à des matériaux généralement très hygroscopiques, ou ayant des températures de ramollissement très basses, de telle sorte que leur vocation matériaux s'en trouve très compromise.

Les chlorures dont l'aptitude à vitrifier à été démontrée sont les suivants :
$-\mathrm{ZnCl}_{2}$, seul ou associé à d'autres chlorures, donne des verres qui ont une bonne transmission jusqu'à $14 \mu \mathrm{m}$

- $\mathrm{BiCl}_{3}$, associé à $\mathrm{KCl}$, permet d'obtenir des verres sans trempe

- $\mathrm{ThCl}_{4}$, associé à $\mathrm{KCl}-\mathrm{NaCl}$, donne des matériaux vitreux dans un large domaine de compositions. Dans ces trois types de verres, $\mathrm{ZnCl}_{2}, \mathrm{BiCl}_{3}, \mathrm{ThCl}_{4}$ jouent le rôle de vitrificateur, et les verres obtenus sont stables vis-à-vis de la cristallisation. Ils ont, pour les derniers, des frontières I.R. reculées vers 16-18 $\mu \mathrm{m}$, mais sont extrêmement corrodés par la vapeur d'eau atmosphérique, ce qui perturbe immédiatement l'absorption multiphonon.

De nouvelles combinaisons récentes, où il est impossible d'attribuer à un des halogénures le rôle de formateur de réseau, ont récemment été synthétisées [3]. Des associations du type $\mathrm{AgCl}-\mathrm{CsCl}-\mathrm{AgI}$ forment des verres ayant une bonne transmission jusqu'à 20-25 $\mu \mathrm{m}$, et présentent une faible hygroscopicité. Le concept de formation de verre dans un tel milieu n'est pas explicite, on peut simplement remarquer que les trois halogénures appartiennent à des types structuraux différents, et que ceci rend difficile la réorganisation ordonnée du liquide au passage liquide-solide.

Des verres de bromures et de iodures à base $\mathrm{ZnBr}_{2}$ ou $\mathrm{CdI}_{2}$ ont été synthétisés. L'addition d'adjuvants divers ne permet pas de diminuer significativement la corrosion de ces matériaux par l'humidité atmosphérique et amène parfois les températures de verre jusqu'à la température ordinaire.

Les verres de fluorures ont été découverts il y a une dizaine d'années à l'Université de Rennes et les recherches sur cette nouvelle classe de matériaux se sont développées de façon intensive compte tenu de leurs applications potentielles dans le domaine du moyen I.R. et des télécommunications.

$\mathrm{Si}$ on exclut les verres de $\mathrm{BeF}_{2}$, toxiques, hygroscopiques, isotypes de $\mathrm{SiO}_{2}$ et n'ayant donc pas de propriétés I.R. particulières, trois nouvelles familles de verre ont été découvertes:

1) les verres à base de fluorures de métaux de transition ou d'aluminium $\mathrm{MF}_{3}$ où $\mathrm{M}=\mathrm{Al}, \mathrm{Cr}, \mathrm{Ga}$, $\mathrm{Fe}, \ldots$

2) les verres à base de fluorure de zirconium $\mathrm{ZrF}_{4}$

3) et les verres à base de fluorures de métaux lourds, généralement multicomposants et dont les constituants sont $\mathrm{ThF}_{4}, \mathrm{InF}_{3}, \mathrm{LnF}_{3} \quad(\mathrm{Ln}=$ terres rares), $\mathrm{BaF}_{2}, \mathrm{ZnF}_{2} \ldots$

Les verres de fluorures de métaux de transition [4] ont été découverts et développés à l'Université du Mans. Leur domaine de transparence dans l'I.R. n'est pas supérieur à celui des verres à base $\mathrm{ZrF}_{4}$ et leur tendance à la cristallisation plus importante. Certaines compositions vitreuses, très riches en ions paramagnétiques, comme $\mathrm{PbMnFeF}_{7}$, présentent par contre d'intéressantes propriétés magnétiques de type verre de spin. 


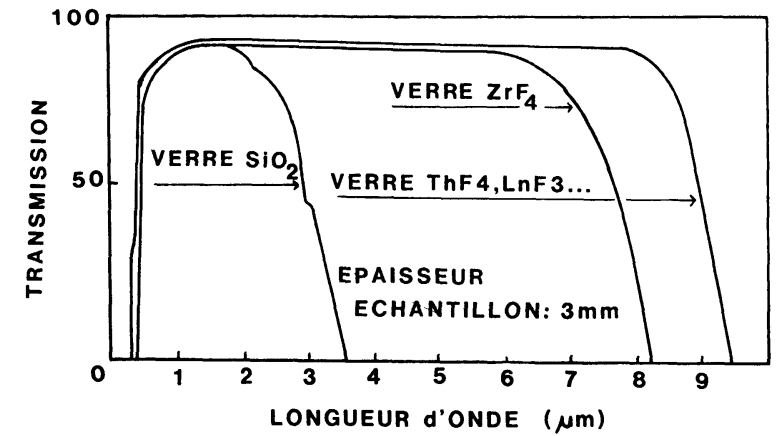

Fig. 2. - Domaine de transmission comparé de verres à base de $\mathrm{SiO}_{2}$, de fluorure de zirconium et de verres multicomposants à base de fluorures de métaux lourds comme $\mathrm{InF}_{3}, \mathrm{ThF}_{4}, \mathrm{BaF}_{2}$, etc...

[Transition domain for some glasses such as $\mathrm{SiO}_{2}$ based, zirconium fluoride based and multicomponent materials, containing heavy metal fluoride such as $\operatorname{InF}_{3}, \mathrm{ThF}_{4}$, $\left.\mathrm{BaF}_{2}, \ldots\right]$

Les verres de fluorure de zirconium sont actuellement les plus étudiés compte tenu de leur faible tendance à la dévitrification. Le domaine de transmission de ces verres est présenté sur la figure 2 , et comparé aux verres à base $\mathrm{SiO}_{2}$ et à l'autre famille de verres à base de fluorures de métaux lourds. L'absorption multiphonon commence à devenir significative vers $7 \mu \mathrm{m}$. La figure 3 montre le diagramme de base à trois composants : $\mathrm{ZrF}_{4}-\mathrm{BaF}_{2}-\mathrm{LaF}_{3}$ où les premiers verres fluorés

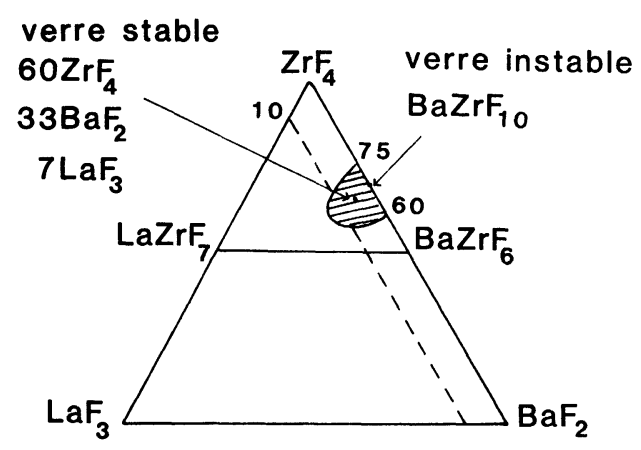

Fig. 3. - Domaine de formation de verres dans le diagramme ternaire $\mathrm{ZrF}_{4}-\mathrm{BaF}_{2}-\mathrm{LaF}_{3}$. Le verre binaire $\mathrm{BaZr}_{2} \mathrm{~F}_{10}$ est sensible à la dévitrification. Le verre $60 \mathrm{ZrF}_{4}, 33 \mathrm{BaF}_{2}, 7 \mathrm{LaF}_{3}$ est plus stable. La composition dite $\mathrm{ZBLA}, 57 \mathrm{ZrF}_{4}, 34 \mathrm{BaF}_{2}, 5 \mathrm{LaF}_{3}, 4 \mathrm{AlF}_{3}$, a une très faible vitesse de dévitrification qui permet, par coulée, d'obtenir des verres de plusieurs centimètres d'épaisseur.

[Glass formation are in the ternary diagram $\mathrm{ZrF}_{4}-\mathrm{BaF}_{2}-\mathrm{LaF}_{3}$. The glass $\mathrm{BaZr}_{2} \mathrm{~F}_{10}$ tends to crystallize easily. The glass $60 \mathrm{ZrF}_{4}, 33 \mathrm{BaF}_{2}, 7 \mathrm{LaF}_{3}$ is more stable. The socalled composition $\mathrm{ZBLA}: 57 \mathrm{ZrF}_{4}, 34 \mathrm{BaF}_{2}$, $5 \mathrm{LaF}_{3}, 4 \mathrm{AlF}_{3}$, has a low crystallization rate which allows the preparation of samples having few centimeters thickness.] ont été découverts. Il existe plusieurs compositions de verres techniques à vitesse de cristallisation réduite, dont le matériau $\mathrm{ZBLA}: 57 \mathrm{ZrF}_{4}, 34 \mathrm{BaF}_{2}$, $5 \mathrm{LaF}_{3}, 4 \mathrm{AlF}_{3}$.

Les verres à base de fluorures de métaux lourds HMFG, Heavy Metal Fluoride Glasses, ne contiennent pas de zirconium et sont des matériaux multicomposants à quatre ou cinq constituants. Deux compositions présentent une stabilité très bonne visà-vis de la dévitrification, autorisant la fabrication de pièces de 1 à $2 \mathrm{~cm}$ d'épaisseur :

1) BTYZ, de composition $16 \mathrm{BaF}_{2}, 28 \mathrm{ThF}_{4}$, $28 \mathrm{YF}_{3}, 28 \mathrm{ZnF}_{2}$

2) BIZYT, de composition $30 \mathrm{BaF}_{2}, 30 \mathrm{InF}_{3}$, $20 \mathrm{ZnF}_{2}, 10 \mathrm{YF}_{3}, 10 \mathrm{ThF}_{4}$.

Ces deux familles de verres ont le même domaine de transparence qui s'étend de $0,2 \mu \mathrm{m}$ dans l'U.V. jusqu'à $8 \mu \mathrm{m}$ dans l'I.R., comme indiqué sur la figure 2 .

2.2 LES VERRES DE CHALCOGÉNURES ET DE CHALCOGĖNE. - La famille des verres de chalcogènes [5] résulte de l'association de chalcogènes entre eux, par exemple des combinaisons binaires ou ternaires des éléments $\mathrm{S}, \mathrm{Se}, \mathrm{Te}, \mathrm{As}, \mathrm{P}$. Les matériaux vitreux comme $\mathrm{As}_{2} \mathrm{~S}_{3}$ et $\mathrm{As}_{2} \mathrm{Se}_{3}$ sont connus depuis longtemps pour avoir une fenêtre de transmission s'étendant de la fin du visible jusqu'à respectivement 12$13 \mu \mathrm{m}$ et $16-17 \mu \mathrm{m}$.

Dans cette série de matériaux, il n'y a pas réellement de verres nouveaux et la tendance actuelle est plus la recherche de combinaisons contenant les éléments les plus lourds pour reculer la frontière I.R. et surtout l'obtention de verres de très grande pureté. La fenêtre optique de ces verres est en effet très facilement altérée par des vibrateurs parasites $\mathrm{S}-\mathrm{O}, \mathrm{S}-\mathrm{H}$, etc... provenant d'impuretés oxygène et hydrogène.

Les verres de chalcogénures résultent des propriétés de formateurs de verres des chalcogénures comme $\mathrm{GeS}_{2}, \mathrm{Ga}_{2} \mathrm{~S}_{3}$ par exemple. Convenablement associés avec d'autres éléments comme Te, $\mathrm{P}, \mathrm{Sb}$, ils donnent des verres à faible vitesse de cristallisation dans de larges domaines de composition. Les systèmes Ge-S, Ge-S-P et leurs équivalents où le Se et le Te remplacent le soufre font encore l'objet de recherche pour obtenir les matériaux les plus transparents dans la région d'émission du laser $\mathrm{CO}_{2}$ à $10,6 \mu \mathrm{m}$.

Les sulfures $\mathrm{GeS}_{2}, \mathrm{Ga}_{2} \mathrm{~S}_{3}$, combinés à d'autres sulfures métalliques jouant le rôle de modificateur de réseau sont plus originaux et donnent ainsi des matériaux ayant une bonne transmission potentielle I.R. dans la région stratégique $8-12 \mu \mathrm{m}$ qui est la deuxième fenêtre de transparence atmosphérique. $\mathrm{Si}$ on associe $\mathrm{GeS}_{2}, \mathrm{Ga}_{2} \mathrm{~S}_{3}$ avec des sulfures de terres rares type $\operatorname{Ln}_{2} \mathrm{~S}_{3}$ ou $\mathrm{MnS}$, on obtient des verres qui sont, comme tous les verres de chalcogénures, sensibles à la corrosion par l'oxygène [6] et leur élaboration doit être effectuée en enceinte scellée. 


\section{Les verres ultratransparents.}

Deux mécanismes intrinsèques sont responsables des pertes quand un matériau est traversé par de la lumière :

1) la diffusion de type Rayleigh qui varie comme $\lambda^{-4}$ et diminue donc quand $\lambda$ augmente

2) l'absorption multiphonon due aux vibrateurs présents dans le matériau, qui augmente avec $\lambda$.

La convergence de ces deux facteurs donne une courbe de pertes en forme de $\mathrm{V}$ qui montre une zone de pertes minima correspondant à un domaine d'ultratransparence du matériau. La figure 4 représente ces courbes de pertes pour des verres à base $\mathrm{SiO}_{2}$ et des verres à base de fluorures.

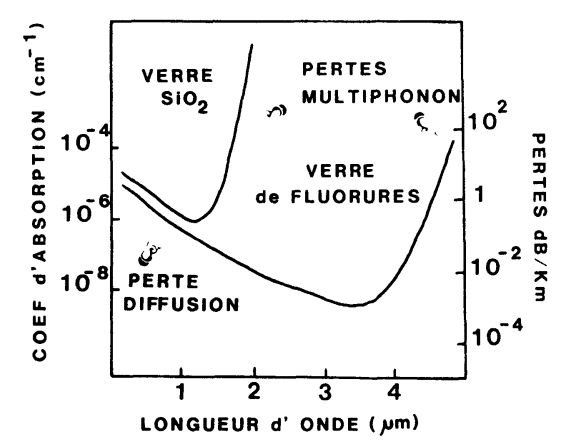

Fig. 4. - Courbe de perte en forme de $\mathrm{V}$ pour des verres à base $\mathrm{SiO}_{2}$ et base fluorures. Les deux mécanismes intrinsèques de pertes, absorption multiphonon et diffusion Rayleigh, évoluent en sens inverse et donnent des pertes minima de $0,2 \mathrm{~dB} / \mathrm{km}$ pour les verres $\mathrm{SiO}_{2}$ et estimées à $10^{-2}, 10^{-3} \mathrm{~dB} / \mathrm{km}$ pour les verres de fluorures.

[The V-shaped loss curve for the $\mathrm{SiO}_{2}$ and fluoride glasses. The intrinsic absorption mechanisms : Rayleigh scattering and multiphonon loss are evoluting in an opposite way versus $\lambda$ and lead to minimum loss of $0.2 \mathrm{~dB} / \mathrm{km}$ for $\mathrm{SiO}_{2}$ glass and estimated $10^{-2}, 10^{-3} \mathrm{~dB} / \mathrm{km}$ for fluoride glasses.]

La transparence ultime théorique, $0,2 \mathrm{~dB} / \mathrm{km}$ vers $1,55 \mu \mathrm{m}$, a été atteinte sur des fibres de $\mathrm{SiO}_{2}$ préparées par des méthodes de dépôt en phase vapeur.

Des pertes estimées de l'ordre de $10^{-2}$, $10^{-3} \mathrm{~dB} / \mathrm{km}$ dans la région $2-5 \mu \mathrm{m}$ sont attendues dans le cas des verres de fluorures. Ce niveau de transparence n'a pas encore été atteint et les pertes minimales mesurées sur des fibres de verre fluoré sont de l'ordre de $3 \mathrm{~dB} / \mathrm{km}$ dans la fenêtre $2,55 \mu \mathrm{m}$ (voir D. C. Tran et S. Takahashi à la Réf. [2]). Ces fibres préparées à partir de procédés classiques : fusion, coulée de préformes doubles indices, puis fibrage, sont encore loin d'être optimisées. Elles contiennent un certain nombre d'imperfections responsables de pertes par diffusion et surtout d'impuretés métalliques absorbant dans cette région du spectre : les ions $\mathrm{Fe}^{2+}, \mathrm{Co}^{2+}, \mathrm{Ni}^{2+}$ et surtout $\mathrm{Nd}^{3+}$ et $\mathrm{OH}^{-}$sont les responsables essentiels des pertes par absorption.

La fenêtre d'ultratransparence la plus réaliste se situera vers $2,55 \mu \mathrm{m}$ entre les métaux de transition et la bande $\mathrm{OH}$ à $2,9 \mu \mathrm{m}$. Toutes les extrapolations montrent que des pertes de $10^{-2} \mathrm{~dB} / \mathrm{km}$, à cette longueur d'onde, sont possibles à condition que le niveau de pertes dues aux ions $\mathrm{OH}$ soit de l'ordre de quelques $\mathrm{dB} / \mathrm{km}$ et que le taux d'impuretés $\mathrm{Nd}^{3+}$ absorbant dans la région $2,5 \mu \mathrm{m}$ soit ramené à des valeurs voisines du centième de ppm.

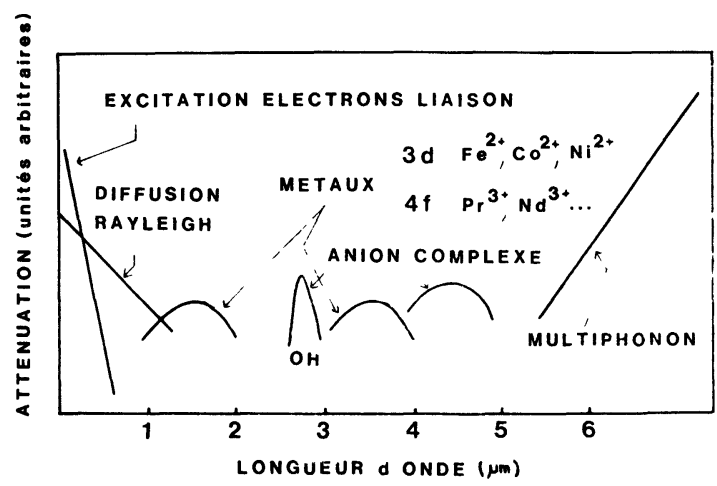

Fig. 5. - En plus des mécanismes intrinsèques, beaucoup d'autres causes extrinsèques sont des facteurs de perte : absorption par les métaux de transition, les terres rares, les anions complexes. A ceci, il faut ajouter des pertes par diffusion dues à des hétérogénéités du matériau : cristallites, séparation de phases, etc...

[In addition to intrinsic mechanisms, extrinsic factors are also responsible of absorption losses : transition metal, rare earth and complexe anions are the most critical. Scattering losses due to inhomogeneities such as microcrystallites, phase separation, ... are also of importance.]

Comme l'indique la figure 5 , les mécanismes de pertes extrinsèques sont multiples dans le moyen I.R., les métaux de transition, les terres rares, les anions complexes sont autant de facteurs d'absorption parasites. A ceci s'ajoute le fait que les verres de fluorures ont une plus forte tendance à la dévitrification que les verres de silice, entraînant la formation de microcristallites et d'imperfections au cours du fibrage, qui entraîne des pertes par diffusion. On estime que, sur les $3 \mathrm{~dB} / \mathrm{km}$ qui constituent le record de transparence actuelle, $2 \mathrm{~dB} / \mathrm{km}$ sont dus à la diffusion et $1 \mathrm{~dB} / \mathrm{km}$ à l'absorption par des impuretés.

Les motivations qui justifient la recherche de matériaux ultratransparents dans le domaine des chalcogénures sont de deux sortes. Comme pour les verres fluorés, l'objectif télécommunications avec des transports de signaux sur de très longues distances, sans répéteurs, est important. Toutefois, les atténuations théoriques ne sont pas plus favorables qu'avec les verres fluorés; de plus, ces matériaux sont très facilement contaminés par des liaisons $\mathrm{S}-\mathrm{H}$ et S-O qui obscurcissent la fenêtre de transparence. 
Il existe toutefois un domaine où la compétition matériaux cristallins- verres est assez équilibrée. Il concerne les guides d'onde I.R. ou fibres I.R. dont la fonction est de transporter des informations I.R. dans la bande la plus large pour la pyrométrie, l'imagerie thermique, la spectroscopie à distance, etc... Les distances sont, dans ce cas, assez courtes et les atténuations exigées quelques $\mathrm{dB} / \mathrm{m}$ peuvent être obtenues avec des fibres polycristallines de type $\mathrm{ZnSe}, \mathrm{KrS}_{5}, \mathrm{AgCl}-\mathrm{AgBr}$ obtenues par extrusion. Dans ce cas, les pertes par diffusion sont toujours importantes, et si on envisage le transport de fortes énergies sur d'assez longues distances, par exemple lors de couplages laser-fibres pour des applications de puissance, l'utilisation de guides d'onde en verre paraît inévitable. Les lasers de puissance disponibles, émettant dans l'I.R., sont assez limités : $\mathrm{Nd}^{3+}$ à $1,06 \mu \mathrm{m}, \mathrm{I}_{2}$ à $1,31 \mu \mathrm{m}, \mathrm{HF}$ à $2,8 \mu \mathrm{m}, \mathrm{DF}$ à $3,8 \mu \mathrm{m}$, $\mathrm{CO}$ à $5,3 \mu \mathrm{m}$ et $\mathrm{CO}_{2}$ à $10,6 \mu \mathrm{m}$. Parmi ceux-ci, le laser $\mathrm{CO}_{2}$ reste un des plus efficaces, ses photons étant fortement absorbés par la matière, ce qui justifie son intérêt dans les domaines de la chirurgie laser, le découpage, la soudure, etc...

La recherche de verres très transparents à cette longueur d'onde pouvant être traversés sans dommage par des énergies importantes reste un objectif fondamental dans beaucoup de centres de recherches matériaux.
Les meilleurs résultats sont de l'ordre de $35 \mathrm{~dB} / \mathrm{km}$ à $2,4 \mu \mathrm{m}$ mesurés sur une fibre cœur-gaine de $\mathrm{As}_{2} \mathrm{~S}_{3}$ [7].

Le second objectif est la réalisation de fibres optiques I.R. suffisamment transparentes à 10,6 $\mu \mathrm{m}$ pour le transport de l'énergie émise par le laser à $\mathrm{CO}_{2}$. Le nombre de verres fibrables transparents dans cette région est très limité et ceux-ci sont obligatoirement à base d'éléments les plus lourds pour que l'absorption multiphonon à $10,6 \mu \mathrm{m}$ soit la plus faible possible.

Actuellement, les résultats les plus significatifs à cette longueur d'onde obtenus sur des fibres de verres sont : $1 \mathrm{~dB} / \mathrm{km}$ pour des compositions à base de $\mathrm{Ge}-\mathrm{Se}-\mathrm{Te}$ [7] et 2 à $3 \mathrm{~dB} / \mathrm{km}$ sur les fibres As-GeSe-Te à double gainage plastique développé aux laboratoires de Marcoussis [8].

\section{Conclusion.}

Le verre, par rapport aux matériaux cristallins qui ont de bonnes propriétés de transmission dans l'infrarouge, présente un avantage certain par sa facilité de mise en forme. En particulier, la réalisation de fibres pour applications télécommunications très longues distances, sans répéteur, nécessite obligatoirement l'utilisation de matériaux vitreux.

\section{Bibliographie}

[1] ZARZYCKI, J., Les verres et l'état vitreux (Masson Ed., Paris) 1982.

[2] Halide Glasses I, II, J. Lucas et C. T. Moynihan Ed., Proceedings of the III Int. Symposium on Halide Glasses, Rennes, June (1985) - Material Science Forum, Trans. Tech. Publications.

[3] ANGEll, C. A., Far I.R. transmitting halide glasses, Mat. Science Forum, 5 (1985) 189-192.

[4] Jacoboni, C., Le Bail, A. et De PAPE, R., Fluoride glasses of 3d transition metals, Glass Technol. 24, 3 (1983) 164-167.
[5] Savage, J. A., Infrared optical materials and their antireflection coatings (Adam Hilger Ltd) 1985.

[6] Barnier, S. Guittard, M. et Flahaut, J., Mat. Res. Bull., 19 (1984) 837.

[7] IwASAKI, H., Proceedings SPIE, I.R. Fibers IV, Los Angeles, Jan. 1986, pap. 618-01.

[8] Wehr, M. C. et Le Sergent, C., Proceedings SPIE, I.R. Fibers IV, Los Angeles, Jan. 1986, pap. 618-19. 